\title{
TU/e emonownen

\section{The vibrational frequency of nitrogen near the fluid-solid transition in the pure substance and in mixtures}

\section{Citation for published version (APA):}

Michels, J. P. J., Kooi, M. E., \& Schouten, J. A. (1998). The vibrational frequency of nitrogen near the fluid-solid transition in the pure substance and in mixtures. Journal of Chemical Physics, 108(7), 2695-2702.

https://doi.org/10.1063/1.475699

DOI:

10.1063/1.475699

Document status and date:

Published: 01/01/1998

\section{Document Version:}

Publisher's PDF, also known as Version of Record (includes final page, issue and volume numbers)

\section{Please check the document version of this publication:}

- A submitted manuscript is the version of the article upon submission and before peer-review. There can be important differences between the submitted version and the official published version of record. People interested in the research are advised to contact the author for the final version of the publication, or visit the $\mathrm{DOI}$ to the publisher's website.

- The final author version and the galley proof are versions of the publication after peer review.

- The final published version features the final layout of the paper including the volume, issue and page numbers.

Link to publication

\section{General rights}

Copyright and moral rights for the publications made accessible in the public portal are retained by the authors and/or other copyright owners and it is a condition of accessing publications that users recognise and abide by the legal requirements associated with these rights.

- Users may download and print one copy of any publication from the public portal for the purpose of private study or research.

- You may not further distribute the material or use it for any profit-making activity or commercial gain

- You may freely distribute the URL identifying the publication in the public portal.

If the publication is distributed under the terms of Article 25fa of the Dutch Copyright Act, indicated by the "Taverne" license above, please follow below link for the End User Agreement:

www.tue.nl/taverne

Take down policy

If you believe that this document breaches copyright please contact us at:

openaccess@tue.nl

providing details and we will investigate your claim. 


\title{
The vibrational frequency of nitrogen near the fluid-solid transition in the pure substance and in mixtures
}

\author{
J. P. J. Michels, M. E. Kooi, and J. A. Schouten \\ Van der Waals-Zeeman Institute, University of Amsterdam, Valckenierstraat 65-67, 1018 XE, Amsterdam, \\ The Netherlands
}

(Received 11 September 1997; accepted 11 November 1997)

\begin{abstract}
At high densities intramolecular vibrations are strongly dependent on the interactions with the surrounding molecules. In this paper a study is made of the consequences of these interactions on the Raman $Q$-branch of nitrogen. In particular the difference between a disordered and an ordered surrounding is surveyed. For this purpose, high-resolution Raman spectroscopy has been performed at room temperature on pure nitrogen as well as on a dilute mixture of nitrogen in argon, around the fluid-solid phase transition of these systems, which occur at $\approx 2.5 \mathrm{GPa}$ and at $\approx 1.3 \mathrm{GPa}$, respectively. Going from the liquid to the solid phase, a positive jump in the line shift and a dramatical drop in the linewidth are seen in both systems at the transition pressure. For a better understanding of the underlying mechanisms, molecular dynamical simulations have been performed on corresponding model systems. The results of these calculations are in fair agreement with the experimental data and reveal the reasons for the discontinuities. Although the average distance of the nearest neighbor molecules around the nitrogen molecule increases, the distance to the nearest neighbor molecules in line with the molecular axis of the nitrogen decrease at the phase transition. This results in a positive jump in the frequency. Further, the time-autocorrelation function of the vibration frequency has a long persisting positive tail in the fluid phase. This behavior is absent in the solid phase. Even more important is that this function has negative values during a substantial time interval in the solid phase. As a result, the correlation time is greatly reduced at the phase transition, which results in an important reduction of the linewidth as well. Finally, it is proven that also in the solid phase the nitrogen is really dissolved in argon. (C) 1998 American Institute of Physics. [S0021-9606(98)50607-1]
\end{abstract}

\section{INTRODUCTION}

During the last years, the behavior of the Raman $Q$-branch of fluid nitrogen as a function of pressure at ambient temperatures has been determined experimentally. ${ }^{1-3}$ Similar experiments have been performed on mixtures of nitrogen with other gases, such as with helium ${ }^{4}$ and, very recently, with argon. ${ }^{5}$ Moreover, it turned out to be possible to simulate the vibrational behavior of nitrogen successfully with the help of molecular dynamical (MD) calculations. ${ }^{6,7}$ In particular, these simulations revealed insight in the details such as correlation times and the influence of the contributions of various mechanisms to the linewidth. Scheerboom and Schouten ${ }^{8}$ measured the Raman shift in three solid phases $(\beta, \delta$, and $\varepsilon)$ of nitrogen and in the fluid phase along isobars, which is relevant for our discussion. Kiefte et al. ${ }^{9}$ and Löwen et al. ${ }^{10}$ give experimental data of the Raman shift for nitrogen in solid matrices of rare gases. Measurements of the latter authors reveal the concentration dependence of the shift at $13 \mathrm{~K}$ and ambient pressure. By extrapolation to zero fraction of nitrogen, the so-called matrix shift has been obtained. Unfortunately one cannot compare these results with the shift in solid pure nitrogen, i.e., the shift of one nitrogen molecule in a matrix of other nitrogen molecules, because of resonance coupling (RC). ${ }^{8}$ At low pressure, this RC coupling is responsible for a continuous behavior of the shift at the fluid-solid $(\beta)$ phase transition. ${ }^{11}$ At high pressure this $\mathrm{RC}$ is probably absent along the $\beta$-melting line. ${ }^{8}$

In this paper, new experimental results are given for the line shift and linewidth of nitrogen in the solid phase and for nitrogen diluted in solid argon, just above the fluid-solid phase transition at ambient temperature and, consequently, at high pressure. It is seen that the solidification at these conditions has a marked influence on the Raman line, also for pure nitrogen. At the phase transition, the line shift increases, while the linewidth decreases considerably. In order to investigate the origin of these effects, MD simulations have been performed on model systems of pure nitrogen in the solid $(\beta)$ phase and of nitrogen infinitely diluted in argon, in the fluid as well as in the solid phase.

\section{METHODS}

\section{A. Experiment}

A full description of the experimental setup has been given elsewhere, ${ }^{5}$ but the most essential facts will be reproduced here. The measurements have been performed in a diamond anvil cell of the membrane type. For the determination of the pressure a small chip of ruby has been put in the cell and the frequency of its luminescence $R 1$ line has been determined. The relation between the frequency and the pressure was taken from the scale of Mao et al. ${ }^{12}$ In order to 
optimize the pressure measurements, the reference wavelength $R 1$ was measured at ambient pressure. An argon-ion laser was used as light source. Further optical components, typically chosen for an optimum efficiency, have been a polarization rotator, a double-monochromator operating in single-pass configuration, and a photomultiplier detection tube. The entrance slit had been fixed to $100 \mu \mathrm{m}$, leading to an instrumental Gaussian profile with a linewidth (FWHM) of about $0.15 \mathrm{~cm}^{-1}$. Before and after each Raman measurement the spectrograph was calibrated using the neon lines at 549 and $553 \mathrm{~nm}$. This resulted in an accuracy of $0.1 \mathrm{~cm}^{-1}$ in absolute wave number. The Raman spectra have been fitted with Lorentzian curves using the least squares approximation method. From these fits the line position and the linewidth have been determined. Finally the deconvolution was performed by substracting quadratically the instrumental FWHM of $0.15 \mathrm{~cm}^{-1}$ from that of the measured profile as described by Ref. 4 . To test the validity of this method, one measured spectrum of solid $\mathrm{N}_{2}$ was deconvoluted with the experimentally determined resolution function. Within experimental accuracy no significant discrepancy has been found between those two methods.

The line shift and line width have been measured at three pressures in solid nitrogen and two measurements have been performed in solid argon, one with 5.9\% $\mathrm{N}_{2}$ and one with $3.0 \% \mathrm{~N}_{2}$.

\section{B. Simulations}

Earlier studies have shown that from computer simulations of pure nitrogen ${ }^{6}$ and dilute mixtures of nitrogen in helium, ${ }^{6,7}$ results can be obtained that are quantitatively in good correspondence with experimental data, notwithstanding the simplifications of the model. The method applied for the calculation of the vibration frequency of nitrogen from molecular dynamics has been described extensively in a previous paper ${ }^{6}$ but, for convenience of the reader, the procedure will be recapitulated briefly below.

For the dynamics the nitrogen particles are modeled as anisotropic rigid rotors; intermolecular forces on the nitrogen molecule consist of forces acting on the nitrogen atoms (sites) of each molecule. From the resulting momentary force $F_{\text {ax }}$ acting on the sites along the molecular axis, called the "external first order effect" $\left(E_{1}\right)$, and from the derivative to the bond length of this force $F^{\prime}$ ax , the "external second order effect" $\left(E_{2}\right)$, the shift of the frequency $d \omega$ with respect to the vibration frequency $\omega_{0 \text {,vib }}$ of an isolated molecule is calculated. Besides, the shift due to the vibrationrotation coupling (VR) is calculated directly from the momentary rotational frequency $\omega_{\text {rot }}$. These three contributions can be calculated from the accurately known intramolecular force constants, experimentally determined by Lavorel et al. ${ }^{13}$ using the theoretical treatment given by Herzberg. ${ }^{14}$ The first- and second-order effect can be regarded as having linear relations with the vibration fre- quency, while the VR is proportional to the rotation frequency squared:

$$
\begin{aligned}
& E_{1}: \quad d_{1} \omega_{\mathrm{vib}}=c_{1} F_{\mathrm{ax}}, \quad c_{1}=3.807 \times 10^{10} \mathrm{~N}^{-1} \mathrm{~cm}^{-1} \\
& E_{2}: d_{2} \omega_{\mathrm{vib}}=c_{2} F_{\text {ax }}^{\prime}, \quad c_{2}=0.507 \mathrm{~N}^{-1} \mathrm{~m} \mathrm{~cm}^{-1}, \\
& \text { VR: } d_{3} \omega_{\mathrm{vib}}=c_{3} \omega_{\text {rot }}^{2}, \quad c_{3}=-3.073 \times 10^{26} \mathrm{~s}^{2} \mathrm{~cm}^{-1} .
\end{aligned}
$$

So far, at least one effect has not been taken into account. At the moment of excitation, the polarizability of the molecule changes and consequently also the interaction energy of the molecule with the surrounding particles. Unfortunately, no accurate values are available for the interaction potential between a nitrogen molecule in the first excited state and surrounding nitrogen molecules in the ground state or with argon atoms. Because this effect is hard to quantify straightforwardly, ${ }^{15}$ an empirical approach has been chosen. The shift, resulting from the three effects mentioned above, is compared with the experimental data. It is assumed that the observed discrepancy is solely due to the omission of the energy jump at excitation. A correction on the potential energy at excitation, called hereafter the "dispersion correction" (DC), is realized by the addition of a Lennard-Jones (LJ) potential $\delta \varphi\left(r_{i, j}\right)$ on both sites (i.e., the atom sites) of the nitrogen molecule. Thus it is assumed that the addition of this correction converts the ground state potential to the potential of an excited molecule. The appropriate parameters for this LJ potential can be found by simulations with which average values $\left\langle r^{-12}\right\rangle$ and $\left\langle r^{-6}\right\rangle, r$ being the distance between interacting sites of different molecules, have been collected for each system as a function of density. The relation between the energy jump $\Delta \varphi$ and the consequent frequency change is given by

$$
\begin{aligned}
& d_{4} \omega_{\text {vib }}=\Delta \varphi / h \mathrm{~cm}^{-1}(h \text { denotes Planck's constant }), \\
& \Delta \varphi=\Sigma \delta \varphi\left(r_{i, j}\right),
\end{aligned}
$$

in which the summation is made over all binary interactions between the atom sites of different molecules.

Although essentially for the red shift of the vibration frequency, the LJ correction potentials, $\delta \varphi\left(r_{i, j}\right)$, resulting in appropriate values of $\Delta \varphi$ are only scanty additions (depending on the intermolecular distance, but always less than $0.6 \%$ ) to the binary intermolecular potentials used for the molecular dynamics. Therefore, these correction potentials have been neglected at the generation of the tracks.

The total frequency change is now defined by

$$
d \omega_{\mathrm{vib}}=\sum_{k=1}^{4} d_{k} \omega_{\mathrm{vib}},
$$

and the Raman shift by

$$
\omega_{\text {shift }}=\omega_{0, \text { vib }}+d \omega_{\text {vib }} .
$$

In here, $\omega_{0, \text { vib }}$ denotes the vibration frequency of an isolated nonrotating molecule. Lavorel et al. ${ }^{13}$ determined experimentally the value at $296 \mathrm{~K}$ :

$$
\omega_{0, \mathrm{vib}}=2329.91 \mathrm{~cm}^{-1} \text {. }
$$




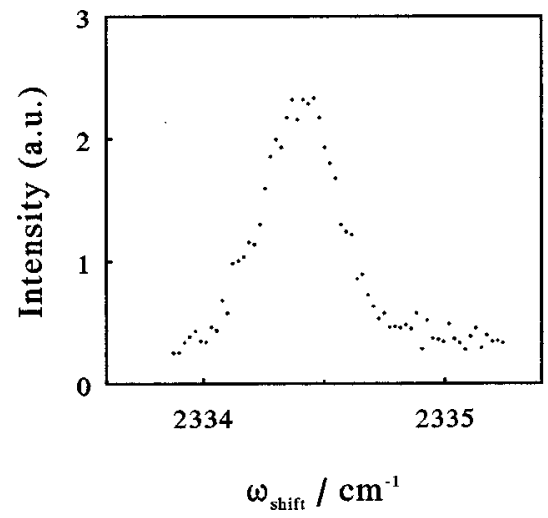

FIG. 1. Example of an experimentally determined Raman line in the solid state of a $5.9 \% \mathrm{~N}_{2}-\mathrm{Ar}$ mixture at $1.9 \mathrm{GPa}$.

After the determination of the parameters needed for the DC, subsequent simulations have been performed. From these runs the following properties, averaged over all particles, have been determined:

(1) The amplitude of modulation $\Delta$, defined as

$$
\Delta=\left\{\left\langle\omega_{\text {vib }}^{2}\right\rangle-\left\langle\omega_{\text {vib }}\right\rangle^{2}\right\}^{1 / 2} \text {. }
$$

(2) The time dependence of the momentary vibrational frequency, in terms of the normalized self-correlation function $\Omega$ and the correlation time $\tau_{c}$ :

$$
\begin{aligned}
& \Omega(t)=\frac{\left\langle\omega_{\mathrm{vib}}(0) \omega_{\mathrm{vib}}(t)\right\rangle-\left\langle\omega_{\mathrm{vib}}(0)\right\rangle^{2}}{\Delta^{2}}, \\
& \tau_{c}=\lim _{t \rightarrow \infty} \int_{0}^{t} \Omega(s) d s .
\end{aligned}
$$

It has been shown by Kubo ${ }^{16}$ in his treatment of the theory of the line shape that the line width can easily be calculated from $\Delta$ and $\tau_{c}$, provided that the system is in the "fast modulation regime." The criterion for fast modulation is given by

$$
\Delta . \tau_{c} \ll 1 .
$$

In that case the line shape will approach the Lorentzian shape with a full line width at half-maximum (FWHM) given by

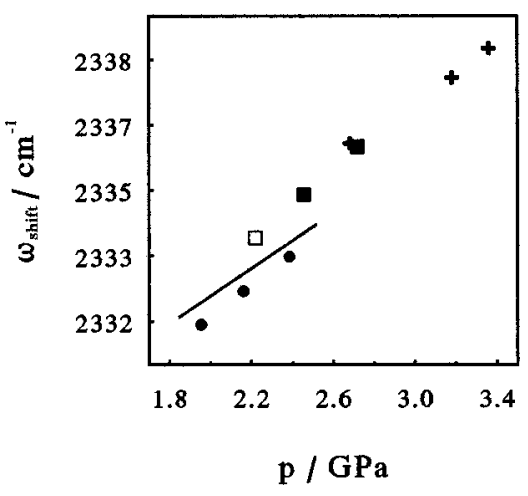

FIG. 2. Raman shift of the $Q$-branch of nitrogen at $296 \mathrm{~K}$ near the fluidsolid phase transition. Line and crosses: experimental results. Dots: MDfluid; squares: MD-solid; open square: metastable state.
TABLE I. Experimental results for the line shift and linewidth of nitrogen and nitrogen in argon, both in the solid phase.

\begin{tabular}{lccc}
\hline \hline $\begin{array}{c}\text { Composition } \\
(\text { mole } \%)\end{array}$ & $\mathrm{P} / \mathrm{GPa}$ & $\omega_{\text {shift }} / \mathrm{cm}^{-1}$ & $\mathrm{FWHM} / \mathrm{cm}^{-1}$ \\
\hline pure $\mathrm{N}_{2}$ & 2.68 & 2336.1 & 0.27 \\
$\ldots$ & 3.18 & 2337.6 & 0.28 \\
$\ldots$ & 3.36 & 2338.3 & 0.27 \\
$3.0 \% \mathrm{~N}_{2}$ in $\mathrm{Ar}$ & 1.71 & 2333.7 & 0.26 \\
$5.9 \% \mathrm{~N}_{2}$ in $\mathrm{Ar}$ & 1.92 & 2334.4 & 0.33 \\
\hline \hline
\end{tabular}

$$
\mathrm{FWHM}=2 \Delta^{2} \cdot \tau_{c} .
$$

In the present study the criterion (9) is checked and the line width is calculated according to relation (10).

\section{RESULTS}

\section{A. Experiment}

Experimental data for the line shift and line width for pure solid nitrogen and for a mixture with $3.0 \%$ and $5.9 \%$ mole fraction of nitrogen in solid argon, at several pressures and at ambient temperature, are given in Table I. It is evident that the intensity of the signals in the dilute mixtures is less than in samples of pure nitrogen. Moreover, the accuracy of the measurement of the line width also decreases with decreasing line width, i.e., in the solid state. Nevertheless, even for dilute mixtures in the solid phase, spectra have been obtained with reasonable quality. As an example, the experimental result for the Raman line of 5.9\% $\mathrm{N}_{2}$ in solid argon is displayed in Fig. 1.

The results for the frequency shift in pure solid nitrogen are given in Fig. 2 by symbols (crosses), and in fluid nitrogen, for which the numbers have been published elsewhere, ${ }^{5}$ by a solid line. Experimental data for the line width in pure nitrogen have been displayed in the same way in Fig. 3: lines for the liquid state, ${ }^{5}$ crosses for the new results concerning the solid phase.

Linear extrapolations of the data to the transition pressure $^{17}(\approx 2.5 \mathrm{GPa})$ reveal a jump in the shift of about $+0.9 \mathrm{~cm}^{-1}$, while the line width (FWHM) decreases with about $0.5 \mathrm{~cm}^{-1}$ to only $1 / 3$ of the fluid value at the transition to the solid phase.

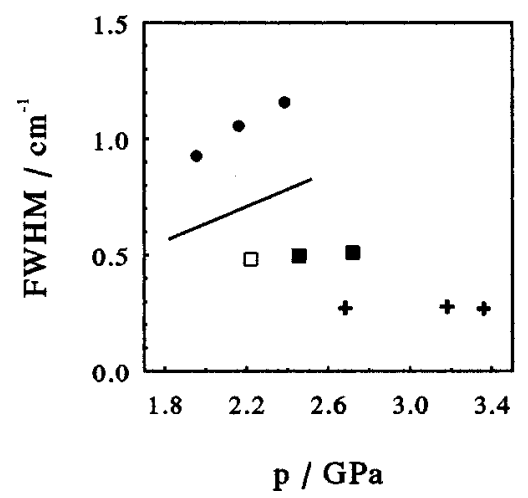

FIG. 3. The linewidth of the Raman $Q$-branch of nitrogen at $296 \mathrm{~K}$ near the fluid-solid phase transition. Line and crosses: experimental results. Dots: MD-fluid; squares: MD-solid; open square: metastable state. 


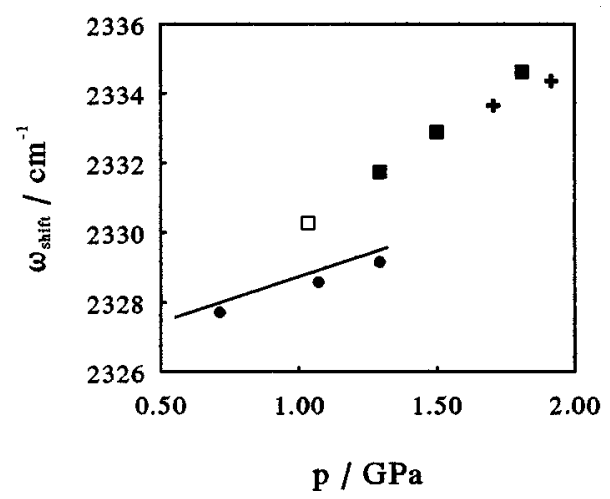

FIG. 4. Raman shift of nitrogen diluted in argon. Lines and crosses: experimental results for mixtures with mole fractions of 0.03 and $0.06 \mathrm{~N}_{2}$. Symbols: MD results for an infinitely diluted mixture of $\mathrm{N}_{2}$ in Ar. Dots: MDfluid; squares: MD-solid; open square: metastable state.

Within experimental errors, measurements ${ }^{5}$ on mixtures of nitrogen in fluid argon gave the same results for the shift and for the line width for concentrations of $5.9 \%$ and $3.0 \%$ nitrogen. Considering the fact that the results even do not differ essentially from the results of pure nitrogen, it is very likely that these numbers also hold for an infinite dilution. The results, including those for the solid phase, have been pictured in Figs. 4 and 5, again indicated with solid lines. An essential question is whether nitrogen dissolves in solid argon. Figure 4 shows a discontinuity in the vibration frequency of the nitrogen molecule, but this could also occur if argon and nitrogen demix. In that case nitrogen is still in the fluid phase. In pure fluid nitrogen, the frequency is 2332.2 $\mathrm{cm}^{-1}$ at $1.9 \mathrm{GPa}$, which is clearly different from 2334.4 $\mathrm{cm}^{-1}$, measured for nitrogen in solid argon. From this one can conclude that nitrogen is dissolved indeed in the crystalline argon. Moreover, only one peak is seen, so the nitrogen must be dissolved completely. Although the uncertainties are larger than in the case of pure nitrogen, one can safely conclude a comparable behavior at the phase transition which occurs at about $1.3 \mathrm{Gpa} .{ }^{17}$ The shift increases with $\approx 1.3$ $\mathrm{cm}^{-1}$ and the linewidth is at least reduced to half, or with roughly $0.3 \mathrm{~cm}^{-1}$ in absolute value.

\section{B. Computational}

\section{Nitrogen near the melting line}

In one of our previous studies ${ }^{6}$ a report was given of simulations of nitrogen in the fluid phase up to the melting line at $296 \mathrm{~K}$, using a site-site potential derived from the

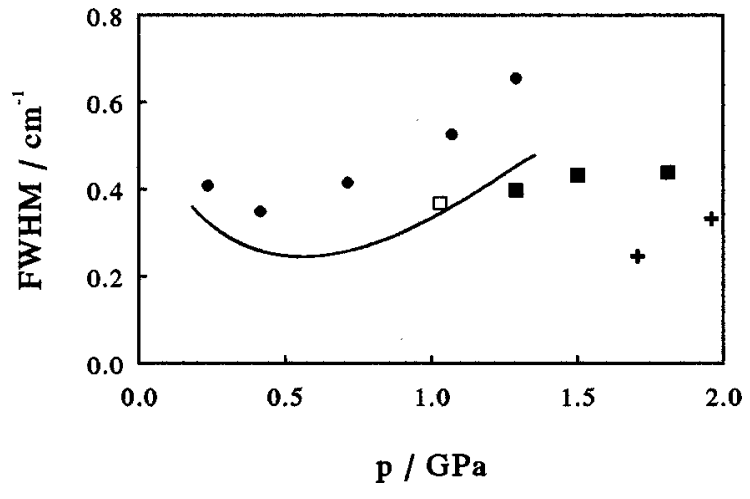

FIG. 5. The linewidth of the Raman line of nitrogen diluted in argon. Lines and crosses: experimental results for mixtures with mole fractions of 0.03 and $0.06 \mathrm{~N}_{2}$. Symbols: MD results for an infinitely diluted mixture of $\mathrm{N}_{2}$ in Ar. Dots: MD-fluid; squares: MD-solid; open square: metastable state.

Etters site-site potential. ${ }^{18}$ In the present work the same model and methods have been applied, but now the calculations have been extended to the solid phase as well. At this temperature, the transition to the solid phase occurs at $\approx 2.5$ GPa. Note that the DC, determined with the help of the experimental data in the fluid phase only, is now also applied in the solid phase. Because solid nitrogen at ambient temperature and just above the transition point is in the $\operatorname{HCP}(\beta)$ phase, ${ }^{19}$ the model system consists of an appropriate rectangular box. The simulations are concerned with 384 particles. Near the transition the system can easily be kept in a metastable state. This enables an overlap for the pressure ranges in the fluid and the solid phase, which may lead to a better insight in the characteristic differences between those states.

Numerical results are given in Table II. A graphical presentation of the shift and of the line width as a function of the pressure are presented in Figs. 2 and 3. For the shift, a good correspondence is seen between the experimental data and the results of the simulations. Also the calculated jump at the melting line is in good agreement with the experiments. The small discrepancy for the shift in the fluid phase is due to the fact that the DC was not determined from the new experimental data but from earlier measurements. ${ }^{3}$ The computations resulted in higher values for the line width in both phases. Nevertheless, the negative jump at the transition is clearly present and, in absolute value, corresponds very well with the experimental behavior. It must be noted that, due to several simplifications, one cannot expect a perfect correspondence. For instance, as mentioned above, the DC has been obtained by the comparison of the calculations with

TABLE II. Results obtained by molecular dynamics for a system of pure nitrogen at $296 \mathrm{~K}$ and pressures near the fluid-solid phase transition.

\begin{tabular}{lccccccc}
\hline \hline State & $\mathrm{P} / \mathrm{GPa}$ & $\rho / \mathrm{kmol}^{-3}$ & $\mathrm{shift} / \mathrm{cm}^{-1}$ & $\Delta / \mathrm{cm}^{-1}$ & $\tau_{c} / \mathrm{ps}$ & $\Delta \tau_{c}$ & $\mathrm{FWHM} / \mathrm{cm}^{-1}$ \\
\hline fluid & 1.956 & 45.0 & 2331.92 & 6.19 & 0.064 & 0.075 & 0.927 \\
fluid & 2.163 & 46.0 & 2332.69 & 6.42 & 0.068 & 0.082 & 1.056 \\
fluid & 2.387 & 47.0 & 2333.48 & 6.62 & 0.070 & 0.087 & 1.157 \\
solid $^{\mathrm{a}}$ & 2.221 & 47.0 & 2333.91 & 6.11 & 0.034 & 0.039 & 0.482 \\
solid & 2.458 & 48.0 & 2334.90 & 6.30 & 0.034 & 0.040 & 0.496 \\
solid & 2.720 & 49.0 & 2336.00 & 6.51 & 0.032 & 0.039 & 0.511 \\
\hline \hline
\end{tabular}

${ }^{\mathrm{a} A n}$ artificial metastable state. 


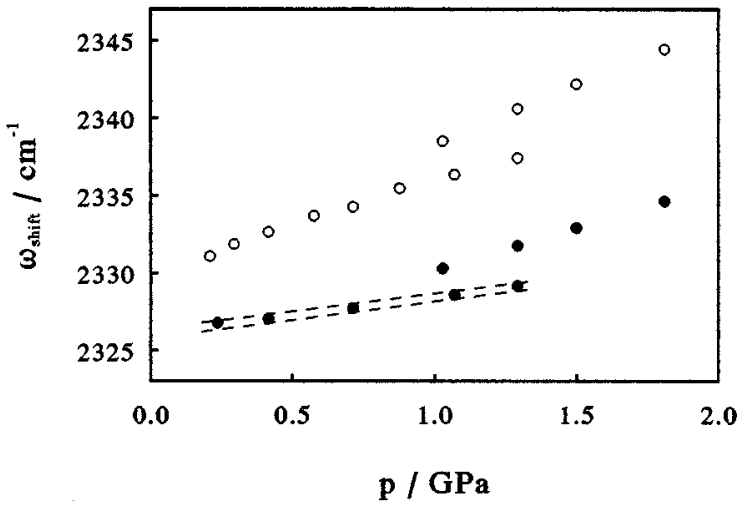

FIG. 6. Raman shift of diluted nitrogen in argon at $296 \mathrm{~K}$ as a function of the pressure. The area between the dashed lines denotes experimental values, ${ }^{18}$ including uncertainties. Open circles: MD results without the dispersion correction (DC); dots: MD results including DC. Results without DC in the fluid range are obtained by short runs only.

the experimental results in the fluid phase only. It is conceivable that the results in the solid phase are more sensitive for the precise shape of the anisotropic interaction potential, including the DC. Also serious may be the omission of the quadrupolar interaction, which has a substantial influence on the crystalline structure. ${ }^{20}$ Notwithstanding these restrictions, the simulations show a good correspondence with the experimental results for the behavior near the phase transition.

\section{Nitrogen in argon near the melting line}

Simulations of mixtures with a small but finite diluted fraction may result in erroneous results due to a suppression of concentration fluctuations in small ensembles. Therefore a system with an infinite dilution has been chose for the cal-
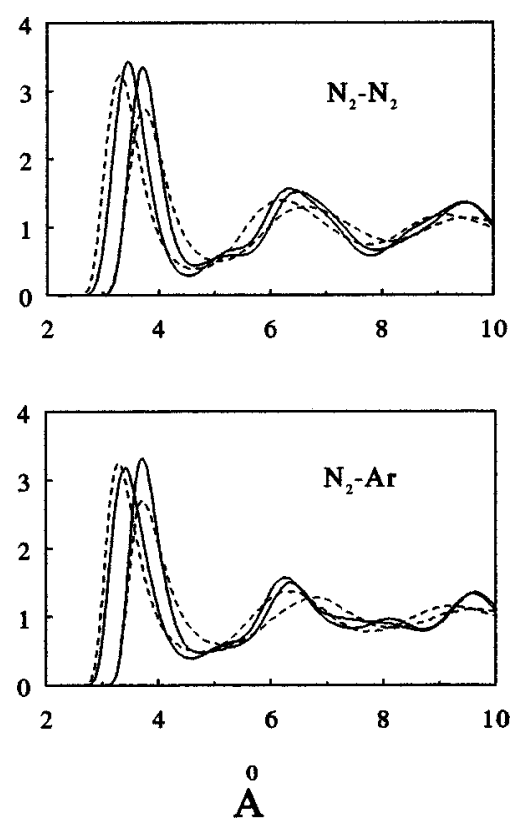

FIG. 7. Pair distribution relative to the center of mass of a nitrogen molecule. Upper figure: in nitrogen at a density of $47 \mathrm{kmol} / \mathrm{m}^{3}$, lower figure: in argon at $48 \mathrm{kmol} / \mathrm{m}^{3}$. Solid line: sold phase (metastable); dashed line: fluid phase. Left curves: perpendicular to the molar axis; right curves: in line with the molar axis. culations. This system consisted of 255 model particles of argon and one for nitrogen in a cubic box, allowing for a fcc structure in the solid phase. For all interactions between the argon molecules as well as for the interaction between an argon molecule and a nitrogen atom (site), exponential-6 potentials have been adopted

$$
\varphi(r)=\frac{\varepsilon}{\alpha-6}\left\{\exp \left[\alpha\left(1-\frac{r}{r_{m}}\right)\right]-\alpha\left(\frac{r_{m}}{r}\right)^{6}\right\},
$$

with the following parameter values:

\begin{tabular}{llll} 
& $\varepsilon / k_{B}(\mathrm{~K})$ & $r_{m}(\AA)$ & $\alpha$ \\
\hline $\mathrm{Ar}-\mathrm{Ar}$ & 122 & 3.85 & 13 \\
$\mathrm{Ar}-\mathrm{N}_{\text {atom }}$ & 69.3 & 3.79 & 13.75 \\
$\mathrm{~N}_{\text {atom }}-\mathrm{N}_{\text {atom }}$ & 39.4 & 3.73 & 14.55 \\
intramolecular & $\mathrm{N}-\mathrm{N}$ distance: & $1.094 \AA$. &
\end{tabular}

Because only one nitrogen molecule is present in the system, the $\mathrm{N}_{\text {atom }}-\mathrm{N}_{\text {atom }}$ interaction is obviously irrelevant for the simulations of this mixture, but the parameters have been applied to calculate the $\mathrm{Ar}-\mathrm{N}_{\text {atom }}$ site interaction using the Lorentz-Berthelot rule for unlike interactions:

$$
\begin{aligned}
& \varepsilon_{1,2}=\left(\varepsilon_{1} \cdot \varepsilon_{2}\right)^{1 / 2}, \\
& r_{m 1,2}=\left(r_{m 1}+r_{m 2}\right) / 2, \\
& \alpha_{1,2}=\left(\alpha_{1} \cdot \alpha_{2}\right)^{1 / 2} .
\end{aligned}
$$

Although this mixing rule describes the unlike interaction only approximately, one may expect that it is appropriate for our purpose. Previous simulations on helium-nitrogen mixtures ${ }^{7}$ strongly indicate that the results for the Raman spectra are rather insensitive for the precise form of the unlike interactions. Moreover, because the statistics had to be taken from the behavior of only one nitrogen molecule, very long simulation runs had to be performed. In view of the efficiency of the program, this simple interaction potential was preferable, although a more realistic potential is available. $^{21}$

First, eight runs have been performed at $T=296 \mathrm{~K}$ and in the pressure range of $0.2 \mathrm{GPa}$ up to the transition pressure, i.e., $1.3 \mathrm{GPa}$, (thus, all in the fluid range), without a dispersion correction in order to determine this correction from comparison with experimental data, as explained above. The necessary cutoff for the interactions was in any case equal to half the cubic box length; the simulations corresponded to a time interval of $2 \times 10^{-10} \mathrm{~s}$. From these simulations, values for $\left\langle r^{-12}\right\rangle$ and $\left\langle r^{-6}\right\rangle$ have been obtained. The effect of the sum of $E_{1}, E_{2}$, and the VR on the vibration frequency has been compared with data obtained from provisional experiments on dilute mixtures of nitrogen in argon. ${ }^{22}$ Although less accurate than the recent results, ${ }^{5}$ these data were already available in an early stage of the project. The LJ parameters for the DC have been calculated from the difference as described in Sec. II:

$$
\begin{aligned}
& \varepsilon / k_{B}=0.89 \mathrm{~K}, \\
& \sigma=2.6 \AA .
\end{aligned}
$$

Figure 6 shows the results of this correction. We emphasize that the values (13) are indicative only. Other combinations 
TABLE III. Results obtained by molecular dynamics for an infinite diluted mixture of nitrogen in argon at $296 \mathrm{~K}$.

\begin{tabular}{lccccccc}
\hline \hline State & $\mathrm{P} / \mathrm{GPa}$ & $\rho / \mathrm{kmol}^{-3}$ & $\omega_{\text {shift }} / \mathrm{cm}^{-1}$ & $\Delta / \mathrm{cm}^{-1}$ & $\tau_{c} / \mathrm{ps}$ & $\Delta \tau_{c}$ & $\mathrm{FWHM} / \mathrm{cm}^{-1}$ \\
\hline fluid & 0.236 & 32.0 & 2326.77 & 3.37 & 0.095 & 0.061 & 0.408 \\
fluid & 0.416 & 37.0 & 2327.05 & 3.78 & 0.065 & 0.046 & 0.349 \\
fluid & 0.714 & 42.0 & 2327.71 & 4.34 & 0.059 & 0.048 & 0.415 \\
fluid & 1.067 & 46.0 & 2328.58 & 4.81 & 0.060 & 0.055 & 0.526 \\
fluid & 1.292 & 48.0 & 2329.16 & 5.10 & 0.070 & 0.064 & 0.655 \\
solid $^{\mathrm{a}}$ & 1.033 & 48.0 & 2330.28 & 4.70 & 0.044 & 0.039 & 0.368 \\
solid $_{\text {solid }}$ & 1.293 & 50.35 & 2331.75 & 5.02 & 0.042 & 0.040 & 0.398 \\
solid & 1.507 & 52.0 & 2332.90 & 5.23 & 0.042 & 0.041 & 0.432 \\
\hline \hline
\end{tabular}

${ }^{\mathrm{a} A n}$ artificial metastable state.

can be found within $\pm 30 \%$ and $\pm 10 \%$ respectively for $\varepsilon$ and $\sigma$ of (13) that give a correspondence between the calculated and experimental data within the estimated accuracy. Nevertheless it has been found that one cannot correct with an $r^{-6}$ or an $r^{-12}$ term only. Five simulation runs have been performed thereupon, corresponding with $2 \times 10^{-9} \mathrm{~s}$, at the same temperature and at pressures ranging from $0.24-1.29$ $\mathrm{GPa}$ (fluid); three simulations in the solid range: 1.29, 1.51, and $1.82 \mathrm{GPa}$; and one in a metalstable solid phase at 1.03 $\mathrm{GPa}$. In the latter simulations, the particles remained ordered in the fcc structure. It is clearly seen that the increase of the length of the runs improves the reproducibility of the data. The final results for the shift just below and above the phase transition are also given in Fig. 4, together with the experimental data. The behavior of the line width as a function of pressure is pictured in Fig. 5 Numerical data are given in Table III. In our previous study on pure nitrogen the addition of the DC increased the line width with about $20 \%$. It is remarkable that in the $\mathrm{N}_{2}-\mathrm{Ar}$ system an overall decrease was seen by about the same percentage. It was seen that the net contribution of the cross terms with the DC to $\Delta$ as well as to $\tau_{c}$ is negative, resulting in a lower value for the line width. A comparison with the experimental results for the jumps in the shift and in the line width leads to the same conclusion as for pure nitrogen: a very good correspondence for the increase of the frequency and a fair correspondence for the line width, although the absolute values for the width are too high, in the fluids as well as in the solid phase.

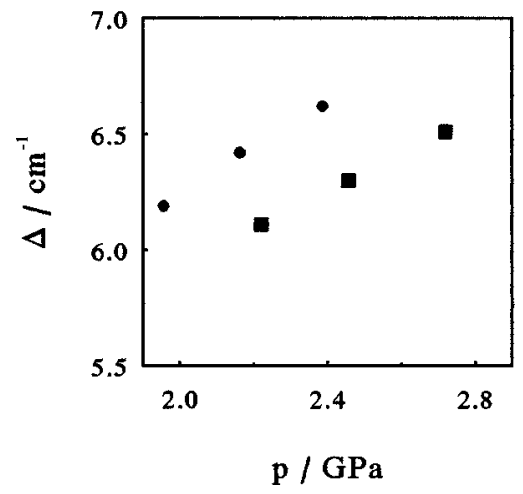

FIG. 8. The amplitude of modulation in the system of pure nitrogen. Dots: fluid; squares: solid.

\section{DISCUSSION}

It is seen that the positive jump in the line shift, experimentally found at the transition to the solid phase, is also clearly present in the simulations, for the system of pure nitrogen as well as for nitrogen in argon. These changes are mainly due to the changes in the first- and second-order effects $E_{1}$ and $E_{2}(1)$, while the proportionality $E_{2} / E_{1}$ remains about 0.2 in both phases for either system. The changes in the contributions of the DC are hardly significant, while the contribution of the vibration-rotation coupling is evidently the same in both phases. It has been noted ${ }^{11,23}$ that the increase of the frequency at the transition to the solid phase is due to the increase of the density. On the other hand, isobaric measurements ${ }^{4}$ and simulations ${ }^{4}$ reveal a constant frequency, suggesting that it is not the density that is primarily responsible for the change in the line shift. In order to solve this question, we performed simulations in both phases, not only at the same pressures, but also at the same densities. Even in the latter case, the frequency in the solid phase turns out to be higher, although the pressure is evidently lower. This effect can be explained qualitatively by a visual analysis of the pair distribution functions. In Fig. 7 the radial distribution function of the centers of mass for nitrogen is given for large angles $[|\cos (\varphi)|<0.1]$ and small angles $[|\cos (\varphi)|>0.9]$ between the line connecting the centers of the molecules and the axis of a central molecule. Note that only the orientation of the central molecule has been taken into account. These results have been obtained for both phases at a density of 47

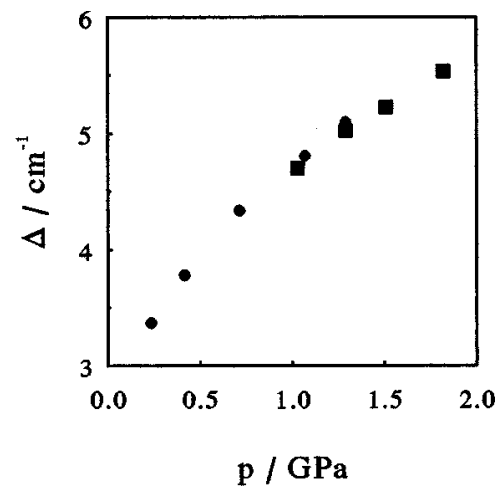

FIG. 9. The amplitude of modulation in the diluted nitrogen-argon system. Dots: fluid; squares: solid. 


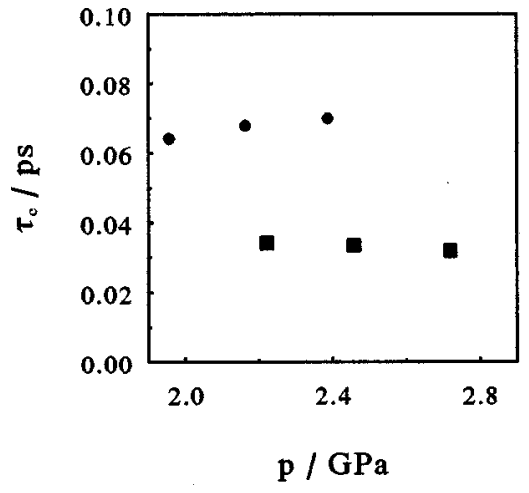

FIG. 10. The correlation time of the vibration frequency of nitrogen near the fluid-solid transition. Dots: fluid; squares: solid.

$\mathrm{kmol} / \mathrm{m}^{3}$. Also in Fig. 7 are given the corresponding distribution functions for argon molecules relative to the center of a single nitrogen molecule at a density of $48 \mathrm{kmol} / \mathrm{m}^{3}$. For both systems and for both phases the nearest neighbors, whose positions are represented by the first maxima in the functions, are in the repulsive region of the molecular interaction, generating the high pressure in the systems. Going from the liquid to the solid phase the first peak of the radial distribution function is shifted to larger distances in comparison with fluid at the same density and, thus, the mean distance between the nearest neighboring particles increases, due to the formation of an ordered structure. This results in a decrease of the repulsive intermolecular forces and, consequently, in a drop of the pressure by ca $0.17 \mathrm{GPa}$ for nitrogen and $0.26 \mathrm{Gpa}$ for argon. This increase in distance is clearly seen for the particles, which are in the solid angles with a large value of $\varphi$. However, the forces of these molecules on the central one have only small components along the molecular axis and therefore have minor influence on the vibration frequency. In contrast, the first maximum in the distribution of the particles, corresponding with small $\varphi$ values, moves even slightly inward and increases considerably in height, giving rise to an increase of the compressive force and consequently cause an increase of the shift.

Also the collapse of the line width at the transition to the solid phase is, at least qualitatively, reproduced by the simulations. According to (10), the line width is determined by the amplitude of modulation $\Delta$ and the correlation time $\tau_{c}$.

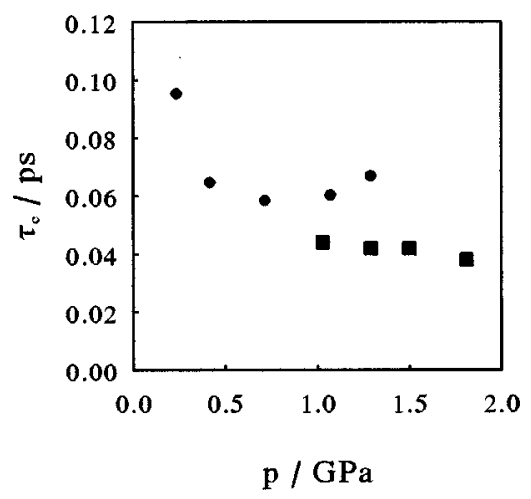

FIG. 11. The correlation time of the vibration frequency of a single nitrogen molecule in argon. Dots: fluid; squares: solid.

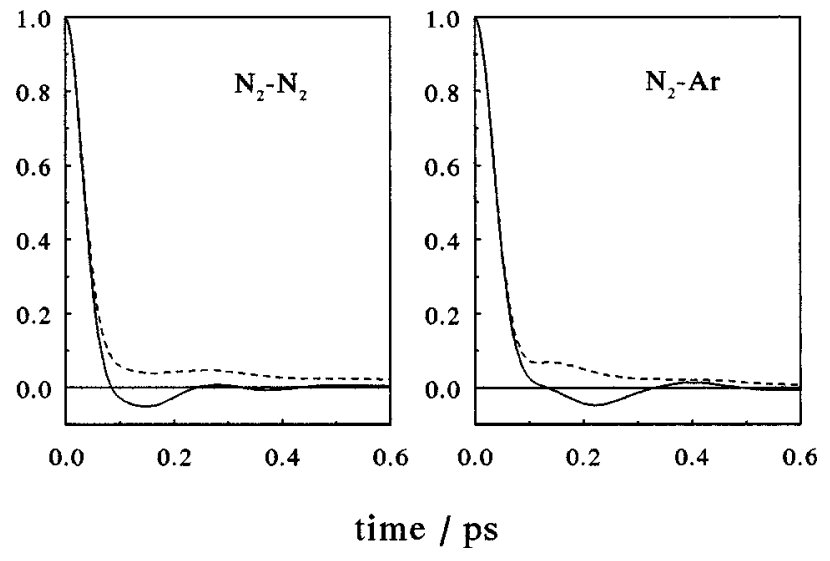

FIG. 12. The initial part of the vibration correlation function of nitrogen in nitrogen at ca $2.4 \mathrm{GPa}$ and of nitrogen in argon at $1.29 \mathrm{GPa}$, both at $296 \mathrm{~K}$. Dashed lines: fluid phase; solid lines: solid phase.

Numbers for these properties are given in the Tables I and II and are depicted in Figs. 8-11. It can be seen directly that the decrease of the line width is for the greater part caused by the behavior of $\tau_{c}: \Delta_{\text {nitrogen }}$ and $\Delta_{\text {argon }}$ decrease only $\approx 6 \%$ and $\approx 2 \%$, respectively, while the correlation time is reduced to about half at the transition. As this correlation time is obtained from the integral of the correlation function $\Omega(t)$ [(7 and 8)], the behavior of the correlation functions deserves more attention. In Fig. 12 the shape of $\Omega(t)$ for the first 0.6 $\mathrm{ps}$ is given for the pure nitrogen system at about $2.4 \mathrm{GPa}$ and for the nitrogen-argon system at $1.3 \mathrm{GPa}$, for the fluid as well as the solid phase. These pictures reveal clearly the cause of the decrease of the correlation times. In the fluid phase, the correlation function drops rapidly until $0.1 \mathrm{ps}$, followed by a long-lasting persistence of positive values. Below ca 0.5 ps, small but reproducible undulations are visible; for longer times the decrease can conveniently be described as an exponential decay. As contrasted with the fluid phase, in the solid phase the correlation functions become negative after ca $0.1 \mathrm{ps}$ and undulate around zero value. After $0.8 \mathrm{ps}$ the values of the functions are no longer significant. In summary, the collapse of the line width is caused primarily by the decrease of the correlation time, which in turn is due to the disappearance of long-time behavior and to the occurrence of negative values of the correlation functions. It is remarkable that this behavior of the correlation function in a solid matrix resembles the results by dynamical simulations in the dilute mixtures of nitrogen in helium ${ }^{7}$ in the fluid phase!

${ }^{1}$ B. Lavorel, B. Oksengorn, D. Fabre, R. Saint-Loup, and H. Berger, Mol. Phys. 75, 397 (1992).

${ }^{2}$ L. Bonamy, J. Bonamy, D. Robert, B. Lavorel, R. Saint-Loup, R. Chaux, J. Santos, and H. Berger, J. Chem. Phys. 89, 5568 (1988).

${ }^{3}$ M. I. M. Scheerboom and J. A. Schouten, Phys. Rev. Lett. 71, 2252 (1993); Phys. Rev. 51, R2747 (1955).

${ }^{4}$ M. I. M. Scheerboom, J. P. J. Michels, and J. A. Schouten, J. Chem. Phys. 104, 9388 (1996).

${ }^{5}$ M. E. Kooi, L. Ulivi, and J. A. Schouten, Int. J. Thermophys. (accepted).

${ }^{6}$ J. P. J. Michels, M. I. M. Scheerboom, and J. A. Schouten, J. Chem. Phys. 103, 8338 (1995); J. Chem. Phys. 105, 9748 (1996).

${ }^{7}$ J. P. J. Michels and J. A. Schouten, Mol. Phys. 91, 253 (1997).

${ }^{8}$ M. I. M. Scheerboom and J. A. Schouten, J. Chem. Phys. 105, 2553 (1996). 
${ }^{9}$ H. Kiefte, M. J. Clouter, N. H. Rich, and S. F. Ahmad, Can. J. Phys. 60, 1204 (1982)

${ }^{10}$ H. W. Löwen, H. J. Jodl, A. Loewenschuss, and H. Däufer, Can. J. Phys. 66, 308 (1987)

${ }^{11}$ R. D. Beck, M. F. Hineman and J. W. Nibler, J. Chem. Phys. 92, 7068 (1990).

${ }^{12}$ H. K. Mao, J. Xu, and P. M. Bell, J. Geophys. Res. 91, 4673 (1986).

${ }^{13}$ B. Lavorel, R. Chaux, R. Saint-Loup, and H. Berger, Opt. Commun. 62, 25 (1987).

${ }^{14}$ G. Herzberg, in Infrared and Raman Spectra of Polyatomic Molecules (Van Nostrand Reinhold, New York, 1962).

${ }^{15}$ D. Levesque, J. J. Weis, and D. W. Oxtoby, J. Chem. Phys. 72, 2744 (1980).

${ }^{16}$ R. Kubo, in Fluctuation, Relaxation and Resonance in Magnetic Systems, edited by D. ter Haar (Oliver and Boyd, Edinburgh, 1962).

${ }^{17}$ L. van der Putten and J. A. Schouten, High Temp.-High Press. 18, 393 (1986).

${ }^{18}$ R. D. Etters, V. Chandrasekar, E. Uzan, and K. Kobashi, Phys. Rev. 33, 8615 (1986).

${ }^{19}$ R. L. Mills, D. H. Liebenberg, and J. C. Bronson, J. Chem. Phys. 63, 1198 (1975).

${ }^{20}$ A. Mulder, J. P. J. Michels, and J. A. Schouten, J. Chem. Phys. 105, 3235 (1996).

${ }^{21}$ L. Benedetti, P. Casavecchia, G. G. Volpi, C. C. K. Wong, and F. W. R. McCourt, J. Chem. Phys. 98, 7926 (1993).

${ }^{22}$ W. D. Koster (unpublished results).

${ }^{23}$ Y. Guissani, D. Levesque, J. J. Weis, and D. W. Oxtoby, J. Chem. Phys. 77, 2153 (1982) 